Anaesthesist 2022 $\cdot 71: 148-149$

https://doi.org/10.1007/s00101-021-01082-8

Angenommen: 8. Dezember 2021

Online publiziert: 3 . Januar 2022

(C) The Author(s), under exclusive licence to Springer Medizin Verlag GmbH, ein Teil von Springer Nature 2022

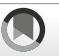

\section{Was ist neu ... bei der Löslichkeit von Fibrinogenpräparaten}

\author{
Matthias Feuerecker ${ }^{1}$. Christa Finkenzeller ${ }^{2}$ \\ ${ }^{1}$ Klinik für Anaesthesiologie, Klinikum der Ludwig-Maximilians-Universität München, München, \\ Deutschland \\ ${ }^{2}$ Abt. Apotheke, Klinikum der Ludwig-Maximilians-Universität München, München, Deutschland
}

\section{Einführung}

Auf dem deutschen Markt sind aktuell 3 Fibrinogenmonopräparate erhältlich, von denen alle eine Zulassung für kongenitale Hypo- oder Afibrinogenämien besitzen [1-3], davon verfügen allerdings nur 2 Produkte zusätzlich über eine Zulassung bei erworbenen Hypofibrinogenämien [1, 2].

Die Präparate werden in unterschiedlichen Grammabpackungen angeboten, sodass seitens der Anwender der Zeitaufwand für die Rekonstitution des Fibrinogenpulvers einen zeitkritischen Faktor darstellen kann.

\section{Das ist neu!}

In den Fachinformationen der beiden Präparate zur Substitution von erworbenem Fibrinogenmangel werden unterschiedliche Angaben zur Zeitdauer des Lösungsvorgangs gemacht: $5-10 \mathrm{~min}$ vs. ca. $5 \mathrm{~min}$ [1, 2].

Seitens der Klinikapotheke wurde das Team der Kardioanästhesie gebeten, das Lösungsverhalten von fibryga ${ }^{\circledR}$ im Vergleich zu Haemocomplettan ${ }^{\circledR}$ (CSL Behring GmbH, Marburg, Deutschland) im Rahmen der Vorbereitung zur intraoperativen Anwendung zu testen. Fibryga ${ }^{\circledR}$ (Octapharma $\mathrm{GmbH}$, Langenfeld, Deutschland) ist ausschließlich in einer Packungsgröße von $1 \mathrm{~g}$ verfügbar, und der Packung ist ein eigenes Transfersystem (Octajet) mit Lösungsmittel beigelegt. Laut Hersteller wird dadurch eine rasche Rekonstitution möglich [2].

Im Rahmen des Vergleichs der beiden Produkte erfolgte u.a. die Zeiterfassung bis zur kompletten Lösung des jeweiligen
Fibrinogenpulvers. Die mitgestoppte Zeit ergab, dass sich fibryga ${ }^{\circledR}$ zumeist nach $31 / 2$ min vollständig gelöst hatte.

Haemocomplettan ${ }^{\circledR}, 1 \mathrm{~g}$ bzw. $2 \mathrm{~g}$ Pulver, benötigte regelhaft mindestens $5 \mathrm{~min}$ bis zur vollständigen Auflösung.

Die im perioperativen Verlauf gemessenen Fibrinogenspiegel beider Produkte zeigten einen adäquaten Anstieg.

Einen „pitfall“ konnten die Autoren bei der Anwendung von fibryga ${ }^{\circledR}$ bisher feststellen: Stellt man die Flasche beim Lösen nicht senkrecht auf einen Tisch, sondern schwenkt sie, z. B. wie im Produktvideo demonstriert [4] bereits während des Transferprozesses, kann der Unterdruck zu früh entweichen, und das Lösen dauert deutlich länger. Aus den bisherigen Beobachtungen ist ersichtlich, dass insbesondere am Ende des Lösungsmitteltransfers das Entweichen des Unterdrucks zu einer raschen Auflösung des Pulvers führt. Dieser Schritt scheint entscheidend zur schnellen Rekonstitution beizutragen.

Anzumerken ist, dass der im Produktvideo zu fibryga ${ }^{\circledR}$ dargestellte Transferprozess nicht der in der Fach- bzw. Gebrauchsinformation angegebenen Vorgehensweise entspricht: „Wenn die Überführung des Lösungsmittels abgeschlossen ist, die Flasche mit dem Arzneimittel vorsichtig schwenken, bis das Pulver vollständig gelöst ist" $[2,5]$.

\section{Fazit für die Praxis}

Mittels des Transfersystems Octajet ist eine schnelle Rekonstitution von fibryga ${ }^{\circledR}$ unter Beachtung der in der Fach-/Gebrauchsinformation angegebenen Arbeitsweise möglich. Eine Vorgehensweise, wie im Produktvideo dargestellt, führt zu einem verzögerten Lösevorgang. 
Obwohl fibryga ${ }^{\circledR}$ nur als $1 \mathrm{~g}$ Präparat verfügbar ist, ist es trotzdem gut geeignet, in der akuten Blutungssituation mit Gerinnungsversagen eine ausreichende Menge an gelöstem Fibrinogen zu generieren, um rasch reagieren zu können.

\section{Korrespondenzadresse}

Prof. Dr. med. Matthias Feuerecker Klinik für Anaesthesiologie, Klinikum der Ludwig-Maximilians-Universität München Marchioninistr. 15, 81377 München, Deutschland

matthias.feuerecker@med.uni-muenchen.de

\section{Dr. Christa Finkenzeller}

Abt. Apotheke, Klinikum der Ludwig-

Maximilians-Universität München

Marchioninistr. 15, 81377 München,

Deutschland

christa.finkenzeller@med.uni-muenchen.de

\section{Einhaltung ethischer Richtlinien}

Interessenkonflikt. M. Feuerecker und C. Finkenzeller geben an, dass kein Interessenkonflikt besteht. Die Autoren haben weder von den genannten Firmen noch von anderen medizinisch, pharmazeutischen Institutionen eine Förderung/Vergünstigung erhalten.
Für diesen Beitrag wurden von den Autoren keine Studien an Menschen oder Tieren durchgeführt. Für die aufgeführten Studien gelten die jeweils dort angegebenen ethischen Richtlinien.

\section{Literatur}

1. CSLBehring (2020)Fachinformation Haemocomplettan ${ }^{\circledR}$ $\mathrm{P} 1 \mathrm{~g} / 2 \mathrm{~g}$

2. Octapharma (2021) Fachinformation Fibryga ${ }^{\circledR} 1 \mathrm{~g}$

3. LFB Biomedicaments (2020) Fachinformation $\mathrm{FibClot}^{\circledR} 1,5 \mathrm{~g}$

4. YouTube (2020) Die korrekte Rekonstitution von fibryga. https://www.youtube.com/watch?v=BN90931rTY.Zugegriffen: 7.Sept. 2021

5. https://www.octapharma.de/api/download/ restricted/cc700f61-d67f-4d3f-8681-0a399fa64380/ fibryga_gebrauchsanweisung-octajet_endversion_ neu.pdf.Zugegriffen:7.Sept. 2021

\section{Hier steht eine Anzeige.}

\section{Springer}

